\title{
Matching and Stabilization of Low-dimensional Nonholonomic Systems
}

Proc. $C D C, \mathbf{3 9},(\mathbf{2 0 0 0 )}, \mathbf{1 2 8 9 - 1 2 9 5 . ~}$

\author{
Dmitry V. Zenkov ${ }^{1}$ \\ Department of Mathematics \\ University of Michigan \\ Ann Arbor, MI 48109 \\ zenkov@math. Isa.umich.edu \\ Naomi E. Leonard \\ Department of Mechanical \\ \& Aerospace Engineering \\ Princeton University \\ Princeton, NJ 08544 \\ naomi@princeton.edu
}

\author{
Anthony M. Bloch ${ }^{2}$ \\ Department of Mathematics \\ University of Michigan \\ Ann Arbor, MI 48109 \\ abloch@math. Isa.umich.edu \\ Jerrold E. Marsden ${ }^{3}$ \\ Control and Dynamical Systems \\ California Institute \\ of Technology 107-81 \\ Pasadena, CA 91125 \\ marsden@cds.caltech.edu
}

\section{Contents}

1 Introduction

2 Overview of Nonholonomic Dynamics

2.1 Equations of Motion of Nonholonomic Systems with Symmetry . . . . . . . . 2

2.2 The Unicycle with Rider . . . . . . . . . 2

\section{Matching and Controlled Lagrangians}

4 Feedback stabilization

\begin{abstract}
In this paper we show how generalized matching technique for stabilization may be applied to the Routhian associated with a low-dimensional nonholonomic system. The theory is illustrated with a simple model - a unicycle with rider.
\end{abstract}

\section{Introduction}

In this paper we apply the method of controlled Lagrangians to the problem of stabilization of steady state motions of low-dimensional nonholonomic systems with

\footnotetext{
${ }^{1}$ Research partially supported by NSF grant DMS-9803181, AFOSR grant F49620-96-1-0100, a University of Michigan Rackham Fellowship, and an NSF group infrastructure grant at the University of Michigan

${ }^{2}$ Research partially supported by NSF grant DMS-9803181, AFOSR grant F49620-96-1-0100, and an NSF group infrastructure grant at the University of Michigan

${ }^{3}$ Research partially supported by AFOSR grant F49620-95-10419
}

.

.

.

,

4

symmetry. The controlled Lagrangian approach for stabilization was introduced in Bloch, Leonard, and Marsden [3, 4] for underactuated holonomic systems with the control force acting along the symmetry directions. Initially the control forces were chosen to preserve symmetry. Later on (see Bloch, Leonard, and Marsden [5] and Bloch, Chang, Leonard, and Marsden [7]) this approach was extended to handle certain types of system with broken symmetry.

The method of Bloch, Leonard, and Marsden requires that specific matching conditions are satisfied. These conditions allow one to introduce a controlled Lagrangian and to rewrite the equations for the controlled (forced) system as the Euler-Lagrange equations for this controlled Lagrangian. Further, the nonlinear controlled inputs derived in this fashion thus become globally defined.

Recently, Auckly, Kapitanski, and White [1] and Hamberg [9] developed a version of this technique for more general systems without symmetry. The matching conditions given by these authors are less restrictive, but the control law is usually local and is harder to obtain (it requires solving a system of PDE's). As in Bloch, Leonard, and Marsden, a control term emulating dissipation is often added to the control input to achieve asymptotic stabilization.

In this paper we consider a class of underactuated nonholonomic systems with two dimensional shape space, no curvature terms in the shape equations, and whose momentum equation has the form of a parallel transport equation. The control is imposed onto one of the shape degrees of freedom and thus is not aligned with the symmetry directions. We use the approach of Auckly, Kapitanski, and White [1] and Hamberg [9] to- 
gether with that of Bloch, Leonard and Marsden [6] to obtain the control law and then use some of the recent theory of nonholonomic mechanics to obtain stability results. Our work here builds on the result of Zenkov, Bloch, and Marsden [16, 17], where we showed that a suitable control input with dissipation (which moves the nonzero part of the spectrum of the linearized equations of motion into the left half plane) plus the use of the so-called Lyapunov-Malkin theorem led to stability.

A key observation in the present paper is that the steady states under consideration for each value of the nonholonomic momentum are dynamically equivalent to the equilibria of an auxiliary holonomic system whose Lagrangian equals the restriction of the Routhian of the original nonholonomic system onto an appropriate momentum level set. Applying holonomic matching techniques to this auxiliary system, we obtain the auxiliary controlled Lagrangian, which is used to construct the controlled Routhian for the nonholonomic system. The equations of motion associated with this controlled Routhian are equivalent to the original controlled equations and thus supply us with a nonlinear control law. The method proposed here gives a systematic procedure for control design in both the linear and the nonlinear settings. The theory is illustrated with a simple model - a unicycle with rider.

We intend in a future publication to consider more general nonholonomic systems, in particular with control inputs acting along some of the symmetry directions as well.

\section{Overview of Nonholonomic Dynamics}

In this section we give a brief exposition of nonholonomic dynamics. We refer the reader to Bloch, Krishnaprasad, Marsden, and Murray [2] and Zenkov, Bloch and Marsden [15] for a complete exposition.

\subsection{Equations of Motion of Nonholonomic Sys- tems with Symmetry}

Symmetries. Suppose we are given a nonholonomic system specified by the Lagrangian $L: T Q \rightarrow \mathbb{R}$ and a (nonintegrable) constraint distribution $\mathcal{D}$. We can then look for a group $G$ that acts on the configuration space $Q$. It induces an action on the tangent bundle $T Q$ and so it makes sense to ask that the Lagrangian $L$ and the distribution $\mathcal{D}$ are invariant. If these properties hold, we say that $G$ is a symmetry group. In many examples the symmetry group will be evident. For instance, for the system considered here, the unicycle with rider, the symmetry group is $S O(2) \times S E(2)$.

Reduced Equations. In this paper we assume that:

1. The shape space $Q / G$ is a smooth two-dimensional manifold.

2. The curvature of the nonholonomic connection is zero.

3. The momentum equation is of the form of a parallel transport equation.

The reduced equation of motion in this case are

$$
\begin{aligned}
\frac{d}{d t} \frac{\partial \mathcal{R}}{\partial \dot{r}^{1}} & =\nabla_{1} \mathcal{R}, & \frac{d}{d t} \frac{\partial \mathcal{R}}{\partial \dot{r}^{2}} & =\nabla_{2} \mathcal{R}+u, \\
\frac{d p_{a}}{d t} & =\mathcal{D}_{a \alpha}^{b} p_{b} \dot{r}^{\alpha}, & a & =1, \ldots, m,
\end{aligned}
$$

where $\mathcal{R}$ is the Routhian, $r^{\alpha}$ are the shape variables, $p_{a}$ are the components of the nonholonomic momentum, and the covariant derivatives in equation (1) are defined by

$$
\nabla_{\alpha}=\partial_{r^{\alpha}}+\mathcal{D}_{a \alpha}^{b} p_{b} \partial_{p_{a}} .
$$

The term $u$ in the shape equations (1) represents the control input. The full dynamics is governed by equations (1) and (2) coupled with the reconstruction equation for the group variables. This reconstruction equation is not needed here as it does not affect the evolution of the shape and the momentum variables, and thus is not used in our stabilization analysis.

The Steady States. The equilibria

$$
r=r_{0}, \quad p=p^{0}
$$

of equations (1) and (2) represent the steady state motions of system. These equilibria are distinguished by the conditions

$$
\nabla_{1} \mathcal{R}=0, \quad \nabla_{2} \mathcal{R}=0
$$

and thus are labeled by the $p^{0}$. We assume that equilibria (4) are unstable in the direction of unactuated shape variable.

\subsection{The Unicycle with Rider}

An example of a nonholonomic system that satisfies assumptions $1-3$ is the unicycle with rider. We model the rider on a unicycle in this paper by a pendulum suspended on a rod attached to the center of a wheel, the rod and the pendulum representing the body and the limb of the rider. The wheel rolls without slipping on a horizontal plane. The pendulum is free to move in the plane orthogonal to the disk, while the rod stays "vertical" in the disk's plane. We view this as a simplified model of a rider on a unicycle in which only the sideways motion of the rider (such as the rider's limbs) is modeled, without pedaling control.

Configuration Space. The configuration space for the unicycle with rider as described above, is $Q=S^{1} \times S^{1} \times$ $S^{1} \times S E(2)$, which we parameterize with coordinates $(\theta, \varkappa, \psi, \phi, x, y)$. As in Figure $1, \theta$ is the tilt of the unicycle itself, $\varkappa$ is that of the limb, and $\psi$ is the angular position of the wheel of the unicycle. The variables $(\phi, x, y)$, regarded as a point in $S E(2)$, represent the angular orientation and position of the point of contact of the wheel with the ground.

The Symmetry Group. This mechanical system is $S O(2) \times S E(2)$-invariant; the group $S O(2)$ represents 


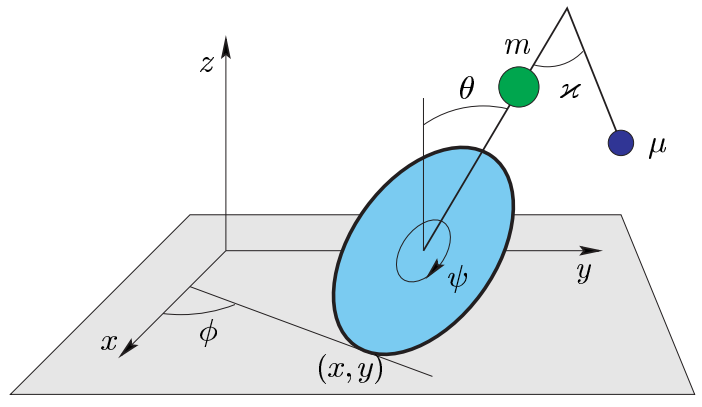

Figure 1: The configuration variables for the unicycle with rider.

the symmetry of the wheel, that is, the symmetry in the $\psi$ variable, while the group $S E(2)$ represents the Euclidean symmetry of the overall system. The action by the group element $(\alpha, \beta, a, b)$ on the configuration space is given by $(\theta, \varkappa, \psi, \phi, x, y) \mapsto(\theta, \varkappa, \psi+\alpha, \phi+$ $\beta, x \cos \beta-y \sin \beta+a, x \sin \beta+y \cos \beta+b)$.

System Parameters. We will use the following notations:

$M=$ the mass of the disk,

$R=$ the radius of the disk,

$A, B=$ the principal moments of inertia of the disk,

$m=$ the rider mass,

$r=$ the rod length,

$l=$ the distance from the center of the disk to the mass $m$,

$\mu=$ the limb mass,

$\rho=$ the limb length.

Reduced Equations. The reduced dynamics of the unicycle is governed by equations (1) and (2) with $r^{1}=\theta$, $r^{2}=\varkappa$, and the Routhian specified below:

$$
\mathcal{R}=\frac{1}{2}\left(g_{11} \dot{\theta}^{2}+2 g_{12} \dot{\theta} \dot{\varkappa}+g_{22} \dot{\varkappa}^{2}-I^{a b} p_{a} p_{b}\right)-U,
$$

where $\left(p_{1}, p_{2}\right)$ is the nonholonomic momentum,

$$
\begin{aligned}
U= & M g R \cos \theta+m g(R+l) \cos \theta \\
& +\mu g[(R+r) \cos \theta-\rho \cos (\varkappa-\theta)] .
\end{aligned}
$$

is the potential energy of the system,

$$
\begin{aligned}
g_{11}= & M R^{2}+m(R+l)^{2} \\
& +\mu\left[(R+r)^{2}-2(R+r) \rho \cos \varkappa+\rho^{2}\right]+A, \\
g_{12}= & \mu\left[(R+r) \rho \cos \varkappa-\rho^{2}\right], \\
g_{22}= & \mu \rho^{2}
\end{aligned}
$$

are the components of the shape metric, and

$$
\begin{aligned}
I_{11}= & A \cos ^{2} \theta+B \sin ^{2} \theta+m(R+l)^{2} \sin ^{2} \theta \\
& M R^{2} \sin ^{2} \theta+\mu[(R+r) \sin \theta+\rho \sin (\varkappa-\theta)]^{2}, \\
I_{12}= & M R^{2} \sin \theta+m R(R+l) \sin \theta \\
& +\mu R[(R+r) \sin \theta+\rho \sin (\varkappa-\theta)]+B \sin \theta, \\
I_{22}= & M R^{2}+m R^{2}+\mu R^{2}+B
\end{aligned}
$$

are the components of the locked inertia tensor. As usual, $I^{a b}$ are the components of the inverse inertia tensor. For the unicycle, $p_{1}$ is the vertical component of the momentum of the system while $p_{2}$ is the component of the disk's momentum along the normal to the disk. See Zenkov, Bloch, and Marsden [15] for details concerning the nonholonomic momenta and the Routhian.

The shape equations for $\left(r^{1}, r^{2}\right)$ describe the motion of the rod and pendulum system, while the momentum equations for $\left(p_{1}, p_{2}\right)$ model the (coupled) wheel dynamics. The coefficients $\mathcal{D}_{a \alpha}^{b}$ in (3) for the unicycle with rider are computed to be

$$
\begin{aligned}
& \mathcal{D}_{11}^{a}=I^{2 a}[\mathcal{A} \cos \theta-\mathcal{B} \cos (\varkappa-\theta)], \\
& \mathcal{D}_{12}^{a}=I^{2 a} \mathcal{B} \cos (\varkappa-\theta), \\
& \mathcal{D}_{21}^{a}=-I^{1 a}[\mathcal{A} \cos \theta-\mathcal{B} \cos (\varkappa-\theta)], \\
& \mathcal{D}_{22}^{a}=-I^{1 a} \mathcal{B} \cos (\varkappa-\theta) .
\end{aligned}
$$

In the above formulae,

$$
\mathcal{A}=M R^{2}+m R(R+l)+\mu R(R+r), \quad \mathcal{B}=\mu R \rho .
$$

See Zenkov, Bloch, and Marsden [15] for the details concerning how one derives and organizes such equations.

\section{Matching and Controlled Lagrangians}

We follow the procedure of Hamberg [9] in our exposition of the generalized matching technique. In the case of linear matching we make use of the work of Bloch, Leonard and Marsden [5, 6].

Consider an underactuated system with the Lagrangian $L: T Q \rightarrow \mathbb{R}$. Suppose that the configuration variables split into two groups $\left(q^{1}, \ldots, q^{m}\right)$ and $\left(q^{m+1}, \ldots, q^{n}\right)$ in such a manner that only the equations corresponding to the second group ${ }^{1}$ are affected by the control forces:

$$
\frac{d}{d t} \frac{\partial L}{\partial \dot{q}^{i}}=\frac{\partial L}{\partial q^{i}}+u_{i}
$$

where $u_{i}$ represent the control inputs and $u_{a}=0$ for $a=1, \ldots, m$. The uncontrolled system has an unstable equilibrium

$$
q=q_{0},
$$

\footnotetext{
${ }^{1}$ One can think of this grouping of generalized coordinates more intrinsically as assuming there is a bundle structure $Q \rightarrow B$, where the fibers of this bundle represent the control directions.
} 
which we want to stabilize using the control inputs. The controlled Lagrangian approach requires a new function $\widetilde{L}$, the controlled Lagrangian, to be constructed such that the equations

$$
\frac{d}{d t} \frac{\partial \widetilde{L}}{\partial \dot{q}^{i}}=\frac{\partial \widetilde{L}}{\partial q^{i}},
$$

are equivalent to (6). We assume that $L$ and $\widetilde{L}$ are of the form

$$
L=\frac{1}{2} g_{i j} \dot{q}^{i} \dot{q}^{j}-U(q), \quad \widetilde{L}=\frac{1}{2} \widetilde{g}_{i j} \dot{q}^{i} \dot{q}^{j}-\widetilde{U}(q),
$$

with $g_{i j}, \widetilde{g}_{i j}, U$, and $\widetilde{U}$ representing the kinetic energy metrics and the potential energies of the initial and the controlled Lagrangians. Following Hamberg, we introduce the tensors:

$$
T_{j k}^{i}=\widetilde{\Gamma}_{j k}^{i}-\Gamma_{j k}^{i}, \quad h_{i}^{j}=g_{i k} \widetilde{g}^{k j}, \quad \widetilde{h}_{i}^{j}=\widetilde{g}_{i k} g^{k j}
$$

where $\Gamma_{j k}^{i}$ and $\widetilde{\Gamma}_{j k}^{i}$ are the Christoffel symbols of the metrics $g_{i j}$ and $\widetilde{g}_{i j}$. The indices $i, j$, and $k$ range from 1 to $n$, and a summation over repeated indices is understood.

The conditions for equivalence of (6) and (8) are called the matching conditions. They generalize the conditions in Bloch, Leonard, and Marsden [3, 4, 5, 6] and are given in the following theorem:

Theorem 3.1. (Hamberg) Equations (6) and (8) are equivalent iff the following two conditions hold for $a=$ $1, \ldots, m$ :

$$
g_{a l} T_{j k}^{l}=0, \quad h_{a}^{j} \frac{\partial \widetilde{U}}{\partial q^{j}}=\frac{\partial U}{\partial q^{a}} .
$$

The explicit formulae for the controls are

$$
u_{\alpha}=\frac{\partial U}{\partial q^{\alpha}}-h_{\alpha}^{j} \frac{\partial \widetilde{U}}{\partial q^{j}}-g_{\alpha l} T_{j k}^{l} \dot{q}^{j} \dot{q}^{k},
$$

$\alpha=m+1, \ldots, n$.

The following theorem gives sufficient conditions for the controlled Lagrangian to exist:

Theorem 3.2. Consider an equilibrium of system (6) with one unactuated degree of freedom $(a=1)$ and suppose that the linearized system is controllable. Then there exists a locally defined matching Lagrangian $\widetilde{L}$ with controlled energy $\widetilde{E}$ having a local minimum at this equilibrium.

The conclusion of this theorem follows from the existence of solution of the Cauchy problem for the system of partial differential equations (10). The details of a proof of a version of this theorem may be found in Auckly, Kapitanski, and White [1].

Theorem 3.2 suggests the following method of construction of the controlled Lagrangian: First, find the solution of the linearized matching conditions that produces a controlled Lagrangian $\mathcal{L}$ for the linearized system with positive definite controlled energy. One can use the technique of Bloch, Chang, Leonard, and Marsden [7] for this linearized matching. Then, using $\mathcal{L}$, produce an initial condition along a noncharacteristic surface of the system of partial differential equations (10) passing through equilibrium (7). Finally, find the (local) solution of (10) and determine the control law. The energy associated to the controlled Lagrangian is positive definite in the neighborhood of the equilibrium (7). The control law (11) thus stabilizes the equilibrium (7).

Of course, the controls provided by Theorem 3.1 cannot accomplish asymptotic stabilization, which can be gained by adding dissipative terms to $u_{\alpha}$, i.e. by controls of the form

$$
u_{\alpha}=\frac{\partial U}{\partial q^{\alpha}}-h_{\alpha}^{j} \frac{\partial \widetilde{U}}{\partial q^{j}}-g_{\alpha l} T_{j k}^{l} \dot{q}^{j} \dot{q}^{k}-d_{\alpha j} \dot{q}^{j} .
$$

For a two degree of freedom system, one can use the following coefficients $d_{\alpha j}$ :

$$
d_{21}=d \widetilde{h}_{1}^{2}, \quad d_{22}=d \widetilde{h}_{2}^{2}, \quad d>0 .
$$

The general procedure of choice of $d_{\alpha j}$ is discussed in Hamberg [9].

Example. As an example, consider a rod and and a pendulum system with the actuator placed on the top of the rod. The Lagrangian of this system may be obtained from (5) by setting $p_{1}=p_{2}=0$. This system is controllable for a generic choice of the system parameters. Therefore, the unstable upward equilibrium of this system can be stabilized by the method of controlled Lagrangians. One can also consider a more general Lagrangian obtained from Routhian (5) by setting $p_{1}=0$. The Euler-Lagrange equations associated with this Lagrangian coincide with equations (1), and the corresponding linearized problem is controllable. According to theorem 3.2, the unstable equilibrium $\theta=0$, $\varkappa=0$ of this system can be stabilized using the matching technique described above. It would be interesting to obtain the corresponding control law explicitly. This issue will be addressed in a future publication.

\section{Feedback stabilization}

In this section we use matching techniques for the stabilization of steady state motions of the nonholonomic systems that were introduced in section 2 . We develop our approach for stabilization of of steady state motions

$$
r=r_{0}, \quad p=p^{0}
$$

that satisfy the following condition:

$$
\nabla_{\alpha} \mathcal{R}_{\mid p=p^{0}}=\partial_{r^{\alpha}} \mathcal{R}_{\mid p=p^{0}} .
$$


Our approach will work equally well if this condition fails, but the exposition in this situation becomes more sophisticated. The general case will be treated in a forthcoming publication.

Controlled Routhian. In order to define the controlled Routhian, we introduce an auxiliary holonomic system whose Lagrangian is

$$
\mathcal{L}_{p^{0}}=\mathcal{R}_{\mid p=p^{0}}
$$

The steady state motion (14) is dynamically equivalent to the equilibrium of the corresponding auxiliary system. We then apply the holonomic matching technique and obtain the controlled Lagrangian $\widetilde{\mathcal{L}}_{p^{0}}$. This involves the new metric $\widetilde{g}_{\alpha \beta}$ and the new potential energy, which we construct in the following form:

$$
\frac{1}{2} I^{a b} p_{a}^{0} p_{b}^{0}+\widetilde{U}(r)
$$

Next, we "unfreeze" the nonholonomic momentum in the above formula. This gives us the controlled amended potential

$$
\widetilde{U}_{a}=\frac{1}{2} I^{a b} p_{a} p_{b}+\widetilde{U}(r)
$$

Finally, we define the controlled Routhian

$$
\widetilde{\mathcal{R}}=\frac{1}{2} \widetilde{g}_{\alpha \beta} \dot{r}^{\alpha} \dot{r}^{\beta}-\widetilde{U}_{a},
$$

the controlled covariant derivatives

$$
\widetilde{\nabla}_{\alpha}=\frac{\partial}{\partial r^{\alpha}}+\widetilde{\mathcal{D}}_{a \alpha}^{b} p_{b} \frac{\partial}{\partial p_{a}},
$$

and introduce the equations

$$
\frac{d}{d t} \frac{\partial \widetilde{\mathcal{R}}}{\partial \dot{r}^{\alpha}}=\widetilde{\nabla}_{\alpha} \widetilde{\mathcal{R}}, \quad \alpha=1,2 .
$$

Observe that the linearization of the controlled equations at equilibrium (14) can be chosen, with appropriate gains, to have pure imaginary spectrum; this follows from theorem 3.2 and our construction.

Nonholonomic Matching. Our main result is the following theorem:

Theorem 4.1. The shape equations (16) associated with the controlled Routhian $\widetilde{\mathcal{R}}$ and the controlled covariant derivatives $\widetilde{\nabla}$ coupled with the momentum equations (2) are equivalent to the original equations (1) and (2) iff the following matching conditions hold:

$$
\begin{aligned}
g_{1 \gamma} T_{\alpha \beta}^{\gamma}=0, \quad h_{1}^{\alpha} \frac{\partial \widetilde{U}}{\partial r^{\alpha}} & =\frac{\partial U}{\partial r^{1}}, \\
h_{1}^{\alpha}\left[\frac{1}{2} \frac{\partial I^{b c}}{\partial r^{\alpha}}+\widetilde{\mathcal{D}}_{a \alpha}^{c} I^{a b}\right] & =\frac{1}{2} \frac{\partial I^{b c}}{\partial r^{1}}+\mathcal{D}_{a 1}^{c} I^{a b} .
\end{aligned}
$$

The control $u$ is given by

$$
u=\nabla_{2} U-g_{2 \beta} \widetilde{g}^{\alpha \beta} \widetilde{\nabla}_{\alpha} \widetilde{U}-g_{2 \gamma} T_{\alpha \beta}^{\gamma} \dot{r}^{\alpha} \dot{r}^{\beta} .
$$

Proof. Note that the momentum equation was not affected when we introduced the shape equations due to $\widetilde{\mathcal{R}}$ and $\widetilde{\nabla}$. The shape equations due to $\mathcal{R}$ are

$$
\begin{aligned}
g_{\alpha \beta} \ddot{r}^{\beta}+\dot{g}_{\alpha \beta}= & \frac{1}{2} \frac{\partial g_{\beta \gamma}}{\partial r^{\alpha}} \dot{r}^{\beta} \dot{r}^{\gamma}-\frac{\partial U}{\partial r^{\alpha}} \\
& -\left[\frac{1}{2} \frac{\partial I^{b c}}{\partial r^{\alpha}}+\mathcal{D}_{a \alpha}^{c} I^{a b}\right] p_{b} p_{c}+u_{\alpha}
\end{aligned}
$$

Solving these for $\ddot{r}^{\beta}$ leads to

$$
\begin{aligned}
\ddot{r}^{\delta}= & g^{\delta \alpha}\left[\frac{1}{2} \frac{\partial g_{\beta \gamma}}{\partial r^{\alpha}} \dot{r}^{\beta} \dot{r}^{\gamma}-\dot{g}_{\alpha \beta}-\frac{\partial U}{\partial r^{\alpha}}\right] \\
& -g^{\delta \alpha}\left[\frac{1}{2} \frac{\partial I^{b c}}{\partial r^{\alpha}}+\mathcal{D}_{a \alpha}^{c} I^{a b}\right] p_{b} p_{c}+g^{\delta \alpha} u_{\alpha} .
\end{aligned}
$$

Similarly,

$$
\begin{aligned}
\ddot{r}^{\delta}= & \widetilde{g}^{\delta \alpha}\left[\frac{1}{2} \frac{\partial \widetilde{g}_{\beta \gamma}}{\partial r^{\alpha}} \dot{r}^{\beta} \dot{r}^{\gamma}-\dot{\widetilde{g}}_{\alpha \beta}-\frac{\partial \widetilde{U}}{\partial r^{\alpha}}\right] \\
& -\widetilde{g}^{\delta \alpha}\left[\frac{1}{2} \frac{\partial I^{b c}}{\partial r^{\alpha}}+\widetilde{\mathcal{D}}_{a \alpha}^{c} I^{a b}\right] p_{b} p_{c} .
\end{aligned}
$$

These equations become equivalent iff we require that (17) and (18) hold.

Nonholonomic Stabilization. In order to stabilize the steady state motion (14), we will use the results of Zenkov, Bloch and Marsden [15] and in particular, the Lyapunov-Malkin theorem (see the Appendix of [15]). First, we choose the values of gains that produce the controlled Routhian that places the four nonzero eigenvalues of the linearization at (14) on the imaginary axis. The remaining $m$ eigenvalues equal 0 .

For the Lyapunov-Malkin theorem to be used, we force all the nonzero eigenvalues to the left half plane. We accomplish that by adding the dissipative control terms (13) to (18):

$$
u=\nabla_{2} U-g_{2 \beta} \widetilde{g}^{\alpha \beta} \widetilde{\nabla}_{\alpha} \widetilde{U}-g_{2 \gamma} T_{\alpha \beta}^{\gamma} \dot{r}^{\alpha} \dot{r}^{\beta}-d \widetilde{h}_{\alpha}^{2} \dot{r}^{\alpha} .
$$

By the Lyapunov-Malkin theorem, the constructed control stabilizes the steady state motion (14) along with nearby steady states. For a "remote" unstable steady state, we just repeat the above construction and obtain a different feedback control law. One can pose the problem of optimal switching between these control laws. This problem is not considered here.

Stabilization of the Unicycle with Rider. Here we apply the above technique to the problem of stabilization of slow vertical steady state motions of the unicycle with rider. These motions are represented by the following one-dimensional manifold of equilibria:

$$
\theta=0, \quad \varkappa=0, \quad p_{1}=0, \quad p_{2}=c .
$$

The slow vertical motions, i.e. those with small values of $c$ are known to be unstable (Zenkov, Bloch, and Marsden [15, 16]. 
As we have mentioned before, property (15) holds for the relative equilibria (20). We thus are able to introduce an auxiliary holonomic system, which is the rod and a pendulum system discussed at the end of section 3 . The equilibrium

$$
\theta=0, \quad \varkappa=0
$$

of this system is unstable. The linearization at this equilibrium is controllable. Therefore our results on nonholonomic stabilization are applicable. The first two groups of the matching conditions (17) are satisfied because of our choice of the controlled Routhian. To satisfy the third group, we set.

$$
\widetilde{\mathcal{D}}_{a \alpha}^{c}=-\frac{1}{2} I_{a b} \frac{\partial I^{b c}}{\partial r^{\alpha}}+\widetilde{h}_{\alpha}^{\gamma}\left[\mathcal{D}_{a \gamma}^{c}-\frac{1}{2} I_{a b} \frac{\partial I^{b c}}{\partial r^{\gamma}}\right] .
$$

With this choice of $\widetilde{\mathcal{R}}$ and $\widetilde{\nabla}_{\alpha}$, the nonlinear control law (19) stabilizes the slow vertical steady state motions of the unicycle.

\section{Appendix}

Consider the system of differential equations

$$
\dot{x}=A x+X(x, y), \quad \dot{y}=Y(x, y),
$$

where $x \in \mathbb{R}^{m}, y \in \mathbb{R}^{n}, A$ is an $m \times m$-matrix, and $X(x, y), Y(x, y)$ represent higher order nonlinear terms.

Theorem. (Lyapunov-Malkin) If all eigenvalues of the matrix $A$ have negative real parts, and $X(x, y)$, $Y(x, y)$ vanish when $x=0$, then the solution $x=0$, $y=0$ of equations $(A)$ is stable with respect to $(x, y)$, and asymptotically stable with respect to $x$. If a solution $(x(t), y(t))$ is close enough to the solution $x=0$, $y=0$, then

$$
\lim _{t \rightarrow \infty} x(t)=0, \quad \lim _{t \rightarrow \infty} y(t)=c .
$$

\section{References}

[1] Auckly, D., L. Kapitanski, \& W. White, [1998] Control of Nonlinear Underactuated Systems. Preprint. update

[2] Bloch, A.M., P.S. Krishnaprasad, J.E. Marsden, \& R. Murray, Nonholonomic Mechanical Systems with Symmetry, Arch. Rat. Mech. An. 136, 1996, 21-99.

[3] Bloch, A.M., N. Leonard, \& J.E. Marsden, Stabilization of Mechanical Systems Using Controlled Lagrangians, Proc. CDC 36, 1997, 2356-2361.

[4] Bloch, A.M., N. Leonard, \& J.E. Marsden, Matching and Stabilization by the Method of Controlled Lagrangians, Proc. CDC 37, 1998, 1446-1451.
[5] Bloch, A.M., N.E. Leonard \& J.E. Marsden, Controlled Lagrangians and the Stabilization of Mechanical Systems I. Trans. IEEE on Autom. Control, 1999, to appear.

[6] Bloch, A.M., N. Leonard and J.E. Marsden, Potential Shaping and the Method of Controlled Lagrangians Proc. CDC 38, 1999, 1652-1657.

[7] Bloch, A.M. D-E. Chang, N.E. Leonard and J.E. Marsden, Controlled Lagrangians and the Stabilization of Mechanical Systems II: Potential Shaping and Tracking. 1999, to appear.

[8] Bloch, A.M., M. Reyhanoglu, \& N.H. McClamroch, Control and Stabilization of Nonholonomic Dynamic Systems, IEEE Trans. Autom. Contr. 37, 1992, 1746-1757.

[9] Hamberg, J., General Matching Conditions in the Theory of Controlled Lagrangians. Proc. CDC 38, 1999, 2519-2523.

[10] Karapetyan, A.V., On Stability of Steady State Motions of a Heavy Solid Body on an Absolutely Rough Horizontal Plane, J. Appl. Math. Mech. 45, 1981, 604608.

[11] Lyapunov, A.M., The General Problem of the Stability of Motion, London, Washington, DC, Taylor \& Francis, 1992.

[12] Malkin, I.G., On the Stability of Motion in the Sense of Lyapunov (in Russian), Mat. Sbornik XXXV, 1938, 47-101.

[13] Markeev, A.P., Dynamics of a Body Being Contiguous to a Rigid Surface (In Russian), Moscow, Nauka, 1992.

[14] Marsden, J.E., Lectures on Mechanics, London Mathematical Society Lecture Note Series 174, Cambridge University Press, 1992.

[15] Zenkov, D.V., A.M. Bloch, \& J.E. Marsden, The Energy-Momentum Method for Stability of Nonholonomic Systems, Dynamics and Stability of Systems, 13, 1998, 123-165.

[16] Zenkov, D.V., A.M. Bloch, \& J.E. Marsden, Stabilization of the Unicycle with Rider. Proc. $C D C \mathbf{3 8}$, 1999, 3470-3471.

[17] Zenkov, D.V., A.M. Bloch, \& J.E. Marsden, The Lyapunov-Malkin Theorem and Stabilization of the Unicycle with Rider, preprint, 2000, Submitted to System and Control Letters. 